\title{
Patient-completed or symptom-based screening tools for endometriosis: a scoping review
}

\author{
Eric Surrey ${ }^{1}$ Cathryn M. Carter ${ }^{2} \cdot$ Ahmed M. Soliman $^{3} \cdot$ Shahnaz Khan $^{4}$. \\ Dana B. DiBenedetti ${ }^{4} \cdot$ Michael C. Snabes ${ }^{3}$
}

Received: 9 February 2017/ Accepted: 25 April 2017/Published online: 25 May 2017

(c) The Author(s) 2017. This article is an open access publication

\begin{abstract}
Purpose The objective of this review was to evaluate existing patient-completed screening questionnaires and/or symptom-based predictive models with respect to their potential for use as screening tools for endometriosis in adult women. Validated instruments were of particular interest.

Methods We conducted structured searches of PubMed and targeted searches of the gray literature to identify studies reporting on screening instruments used in endometriosis. Studies were screened according to inclusion and exclusion criteria that followed the PICOS (population, intervention, comparison, outcomes, study design) framework.

Results A total of 16 studies were identified, of which 10 described measures for endometriosis in general, 2 described measures for endometriosis at specific sites, and 4 described measures for deep-infiltrating endometriosis. Only 1 study evaluated a questionnaire that was solely patient-completed. Most measures required physician, imaging, or laboratory assessments in addition to patientcompleted questionnaires, and several measures relied on complex scoring. Validation for use as a screening tool in adult women with potential endometriosis was lacking in
\end{abstract}

Cathryn M. Carter

ccarter@rti.org

1 Colorado Center for Reproductive Medicine, Lone Tree, CO, USA

2 RTI Health Solutions, 3005 Boardwalk Street, Suite 105, Ann Arbor, MI 48108, USA

3 AbbVie, North Chicago, IL, USA

4 RTI Health Solutions, Research Triangle Park, NC, USA all studies, as most studies focused on diagnosis versus screening.

Conclusions This literature review did not identify any fully validated, symptom-based, patient-reported questionnaires for endometriosis screening in adult women.

Keywords Endometriosis · Patient-reported - Screener · Self-administered $\cdot$ Symptoms

\section{Introduction}

Endometriosis is a painful, inflammatory condition characterized by the development of endometrial-like tissue outside the uterus [1]. Endometriotic lesions may occur at various anatomic sites, including the pelvic peritoneum and the ovary [2]. Deep-infiltrating endometriosis occurs in the pelvic structures below the surface of the peritoneum. More rarely, endometriosis lesions of the bladder, ureter, or extrapelvic sites may also occur [2].

An estimated $10 \%$ of women of reproductive age are affected by endometriosis [3]. Endometriosis causes considerable clinical, economic, and humanistic burden. Clinical symptoms include chronic pelvic pain, dysmenorrhea, and infertility [3], and endometriosis may increase a woman's risk of cancer or autoimmune disorders [4, 5]. Numerous studies have demonstrated the considerable economic burden associated with endometriosis [6-8]. Hospitalizations, especially those related to surgical intervention, are a primary direct cost driver for endometriosis $[6,7,9,10]$. Moreover, endometriosis has a significant social and psychological impact on the lives of women across several domains, including quality of life, intimate relationships, fertility, education and work, and emotional well-being [11, 12]. 
Many women with endometriosis experience delayed diagnosis [13], on average 6-12 years after initially presenting with symptoms [14]. The clinical presentation of endometriosis is variable, and symptoms may overlap with those of other common conditions (e.g., irritable bowel syndrome or interstitial cystitis) [15], making differential diagnosis challenging. Thus, surgical diagnosis, via laparoscopy or laparotomy, is the only definitive means of diagnosing endometriosis [2].

Patient engagement may be key for the effective identification and management of endometriosis. Endometriosis outcomes are subjective, and although pelvic pain is a common symptom, pain alone may not be adequate to discriminate between women with and without endometriosis [16]. A patient-completed, symptom-based screening tool designed to allow women to self-identify potential symptoms of endometriosis could facilitate the initial discussions between patients and physicians, with the potential to reduce diagnostic delay and encourage earlier treatment of endometriosis. The objective of this review was to identify and evaluate the adequacy of existing patient-completed endometriosis screening questionnaires for adult women; symptom-based predictive models with the potential for use as endometriosis screening tools also were evaluated. Studies that reported validation and performance data were of particular interest.

\section{Methods}

In April 2016, we conducted a structured search of the literature indexed in PubMed (via the National Library of Medicine Gateway) using prespecified, reproducible criteria to identify studies reporting on screening instruments used in endometriosis. No date restrictions were applied in the searches. A combination of medical subject heading terms and free-text terms was used in the searches (Table 1). Additional literature was identified through targeted searching of sources such as proceedings of scientific congresses (i.e., American Society for Reproductive Medicine, Society for Reproductive Investigation, American Congress of Obstetricians and Gynecologists, and World Congress of Endometriosis); clinical trial registries; practice guidelines; and the Journal of Endometriosis and Pelvic Pain Disorders, which was not indexed in PubMed at the time of the search. Studies were screened according to predefined inclusion and exclusion criteria that followed the PICOS framework (Table 2). Studies of patient-completed screening tools and/or symptom-based predictive models were included; studies involving diagnosis based solely on surgical findings, imaging, or biomarkers were not the focus and were excluded.

\section{Results}

\section{Literature search results}

Figure 1 presents the results of the literature search and screening. A total of 16 relevant studies were identified for inclusion, of which 12 were indexed in PubMed, 2 were identified via review of titles and abstracts in the Journal of Endometriosis and Pelvic Pain Disorders, 1 was an abstract, and 1 was identified through targeted internet searching.

\section{Evaluation of included studies}

Table 3 summarizes the identified studies, ten of which described measures for endometriosis in general, two of which described measures for endometriosis at specific sites (bladder and rectovaginal), and four of which described measures for deep-infiltrating endometriosis. The types of measures varied, as did their clinical utility. No follow-up studies that used any of the instruments or applied any of the criteria from the identified studies were located. Only one study evaluated a purely patient-completed screening questionnaire [17]; all other studies reported on hybrid measures consisting of patient-completed, clinician-completed, imaging, and/or laboratorybased assessments to predict diagnosis.

Studies of endometriosis, not focused on a specific site

Three studies described measures to identify probable endometriosis or endometriosis-related symptoms [17-19]. Forman et al. [17] developed a 7-point patient-completed questionnaire to differentiate women with a healthy pelvis from women with endometriosis based on patient symptoms (i.e., period pain, pelvic pain unrelated to menstruation, dyspareunia, and vaginal discharge) and medical history (i.e., past use of an intrauterine device, previous laparotomy, and nulligravida). Severe period pain (dysmenorrhea) was the only symptom found to be predictive of endometriosis, and the questionnaire used in the study did not sufficiently differentiate women with endometriosis from women with a normal pelvis. Fasciani et al. [18] developed a literature-based Endometriosis Index-which included 38 variables and parameters derived from the patient pain evaluation, physician consultation, and diagnostic evidence - to predict the presence of endometriosis in general and by site (i.e., peritoneal, ovarian, or deepinfiltrating endometriosis). Although the measure showed potential utility as a noninvasive screening tool to detect endometriosis and differentiate among disease severities, it was not entirely patient-completed and relied on a 
Table 1 Final PubMed search strategy, conducted April 5, 2016 (limits: humans; no comments or editorials)

\begin{tabular}{|c|c|c|}
\hline $\begin{array}{l}\text { Search } \\
\text { number }\end{array}$ & Search terms & $\begin{array}{l}\text { Number of } \\
\text { results }\end{array}$ \\
\hline \multicolumn{3}{|c|}{ Disease terms } \\
\hline 1 & $\begin{array}{l}\text { "Endometriosis"[Majr] OR endometriosis[Title] OR endometrioses[Title] OR endometrioma[Title] OR } \\
\text { endometriomas[Title] OR endometrial lesion*[Title] Limits: English }\end{array}$ & 13,101 \\
\hline \multicolumn{3}{|c|}{ Screening instruments } \\
\hline 2 & 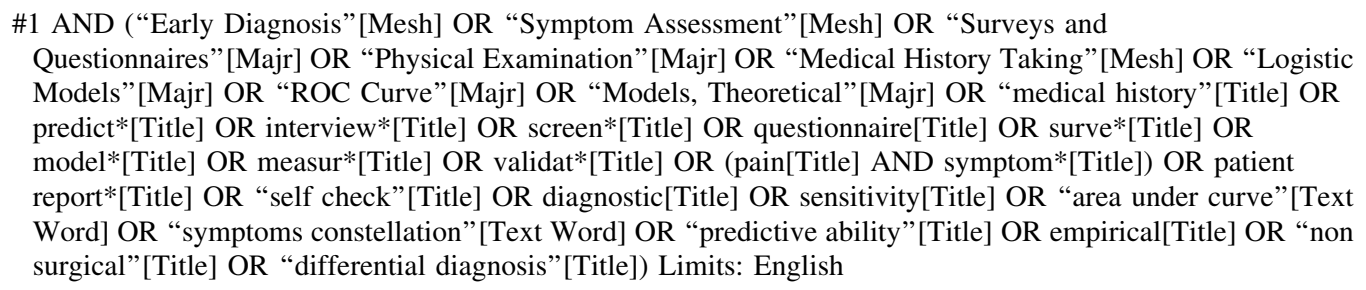 & 881 \\
\hline \multicolumn{3}{|c|}{ Exclusions } \\
\hline \#3 & “Animals"[Mesh] NOT “Humans”[Mesh] Limits: English & $3,745,995$ \\
\hline \#4 & "Comment”[Publication Type] OR "Editorial”[Publication Type] Limits: English & 862,127 \\
\hline$\# 5$ & "Endometriosis/drug therapy"[Mesh] & 1888 \\
\hline \#6 & \#2 NOT (\#3 OR \#4 OR \#5) & 611 \\
\hline
\end{tabular}

Table 2 PICOS inclusion and exclusion criteria

\begin{tabular}{|c|c|c|}
\hline Criteria & Included & Excluded \\
\hline Population & $\begin{array}{l}\text { Studies in women with symptoms consistent with } \\
\text { endometriosis }\end{array}$ & $\begin{array}{l}\text { Studies involving only surgical, imaging, or biomarker } \\
\text { diagnosis of endometriosis }\end{array}$ \\
\hline $\begin{array}{l}\text { Interventions } \\
\text { and } \\
\text { comparators }\end{array}$ & $\begin{array}{l}\text { No specific drug interventions or comparators were the focus } \\
\text { of this review }\end{array}$ & $\begin{array}{l}\text { Studies examining drug interventions in women with a } \\
\text { diagnosis of endometriosis }\end{array}$ \\
\hline Outcomes & $\begin{array}{l}\text { Symptom-based patient-completed endometriosis screening } \\
\text { instruments (questionnaires and/or predictive models) }\end{array}$ & $\begin{array}{l}\text { Instruments other than symptom-based, patient-completed } \\
\text { endometriosis screening questionnaires (e.g., EPBD, ESD, } \\
\text { B\&B) and/or predictive models }\end{array}$ \\
\hline Study design & $\begin{array}{l}\text { Studies of any design that evaluated patient-reported } \\
\text { screening of endometriosis }\end{array}$ & Commentaries and editorials \\
\hline
\end{tabular}

$\overline{B \& B}$ Biberoglu and Behrman, EPBD Endometriosis Pain and Bleeding Diary, ESD Endometriosis Symptom Diary, PICOS population, intervention, comparison, outcomes, study design

comprehensive set of diagnostic parameters including pelvic examination, imaging, and laboratory tests. Yeung et al. [19] developed a predictive mathematical model for the early stage endometriosis based on variables from a preoperative questionnaire that was similar but not identical to the World Endometriosis Research FoundationWomen's Health Symptom Survey (WERF-WHSS). The final model included five factors (patient had low back pain that got worse with periods, but patient had not taken opioids for pelvic pain; body mass index $>39$; patient had period pain affecting daily life and crampy, "period-like" pain without bleeding; patient had crampy, "period-like" pain without bleeding, but did not have dysuria; patient had superficial dyspareunia but not known subfertility). The model was able to differentiate women with endometriosis from those without (AUC $=0.822, P<0.001$; sensitivity $=80.5 \%$; and specificity $=57.7 \%$ ); however, a better specificity would be preferred and it is not feasible as a simple self-completed measure given its complex scoring.

Five studies described presurgical or prelaparoscopic predictive measures specifically [20-24]. Eskenazi et al. [20] aimed to determine whether surgical diagnosis of endometriosis could be predicted via structured patient interviews regarding medical history and symptoms, pelvic examination, and ultrasound findings. Both ultrasound and pelvic examination were $100 \%$ successful in predicting ovarian endometriosis; the other noninvasive procedures were moderately successful in predicting ovarian endometriosis but predicted nonovarian endometriosis less reliably. The presence of any symptom (dysmenorrhea, 


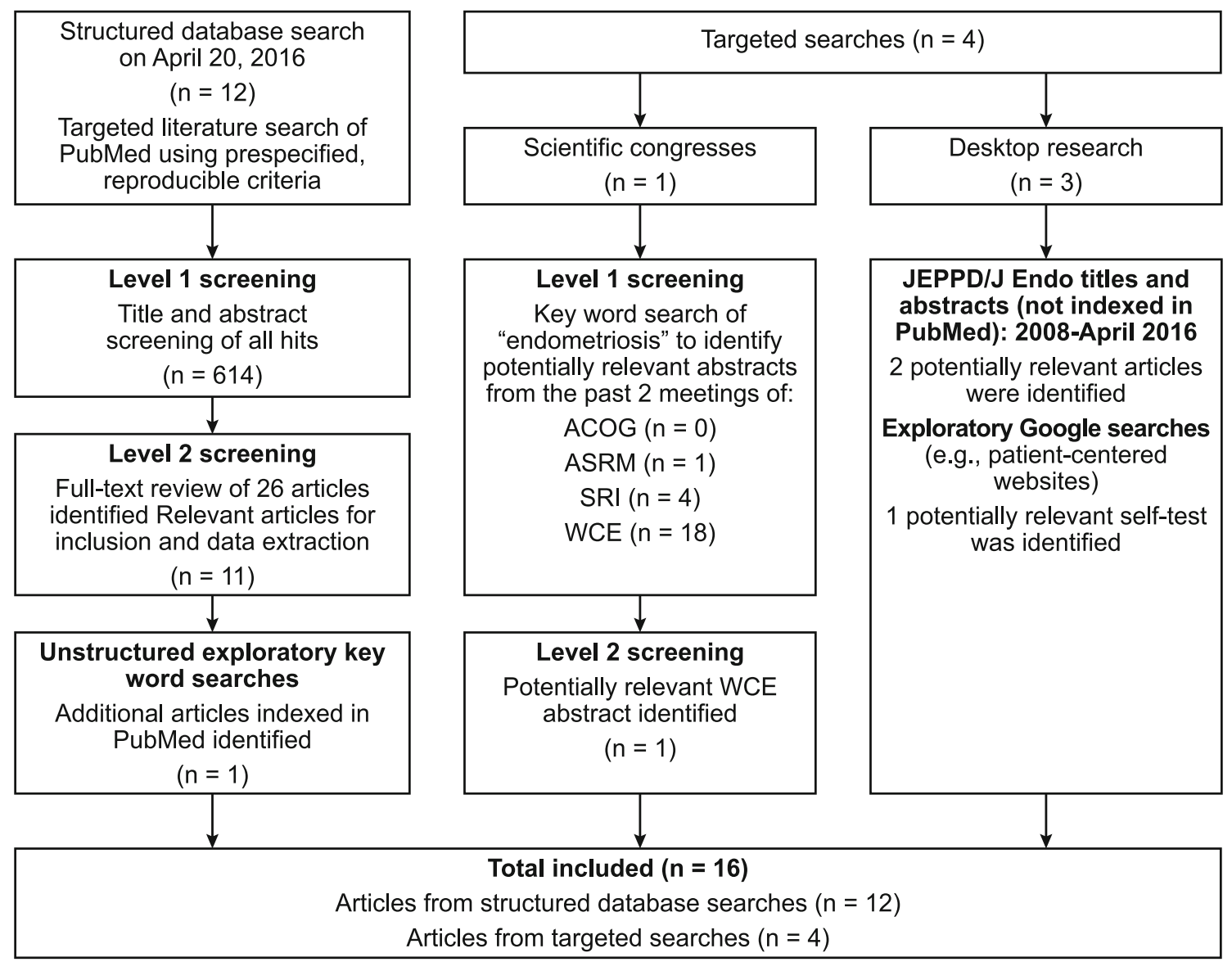

Fig. 1 Literature search results. ACOG, American Congress of Obstetricians and Gynecologists; ASRM, American Society for Reproductive Medicine; JEPPD/J Endo, Journal of Endometriosis

pelvic pain, dyspareunia, or infertility) correctly classified $66 \%$ of endometriosis diagnoses (ovarian and nonovarian combined), with lower positive predictive ability than a positive ultrasound (kappa statistics of 0.32 vs. 0.58, respectively). Symptoms, particularly dysmenorrhea, were more successful in diagnosing ovarian endometriosis than nonovarian endometriosis; therefore, the clinical utility of the symptoms-based approach in this study may be limited for identifying nonovarian endometriosis based on the results of the study by Eskenazi et al. [20].

Calhaz-Jorge et al. [21] developed a mathematical model to predict endometriosis in subfertile women based on medical history and symptoms variables, and collected via personal interview using a standard questionnaire. The variables included age at laparoscopy, weight, height, race, education, lifestyle/smoking habits, obstetric history, duration of subfertility, oral contraceptive use, age at menarche, average duration of bleeding, average cycle length, and the presence and intensity of dysmenorrhea, dyspareunia, and pelvic pain. Primary subfertility, dysmenorrhea, chronic pelvic pain, oral contraception use (ever), and obesity (inverse relationship) were found to be and Pelvic Pain Disorders; SRI, Society for Reproductive Investigation; WCE, World Congress of Endometriosis

predictive of endometriosis. The authors concluded that their findings could be useful for clinicians managing subfertility to help determine when laparoscopy should be performed during the process of managing subfertility; however, the study did not exclude patients with the previous pelvic surgery and was not validated beyond the study population consisting of subfertile, Portuguese women.

Ballard et al. [22] investigated whether different dimensions of chronic pelvic pain are useful in the diagnosis of endometriosis before laparoscopy. They administered a questionnaire evaluating 40 pain descriptors to evaluate descriptions, areas, and intensity of pain, and observed differences in pain dimensions between women with endometriosis and those without, as well as between women with deep versus superficial endometriosis. Dyschezia was more likely to occur in women with endometriosis than in women without endometriosis and was more likely to occur in women with deep endometriosis than in women with superficial endometriosis, and women with endometriosis were also more likely to report their pain as throbbing or gnawing 


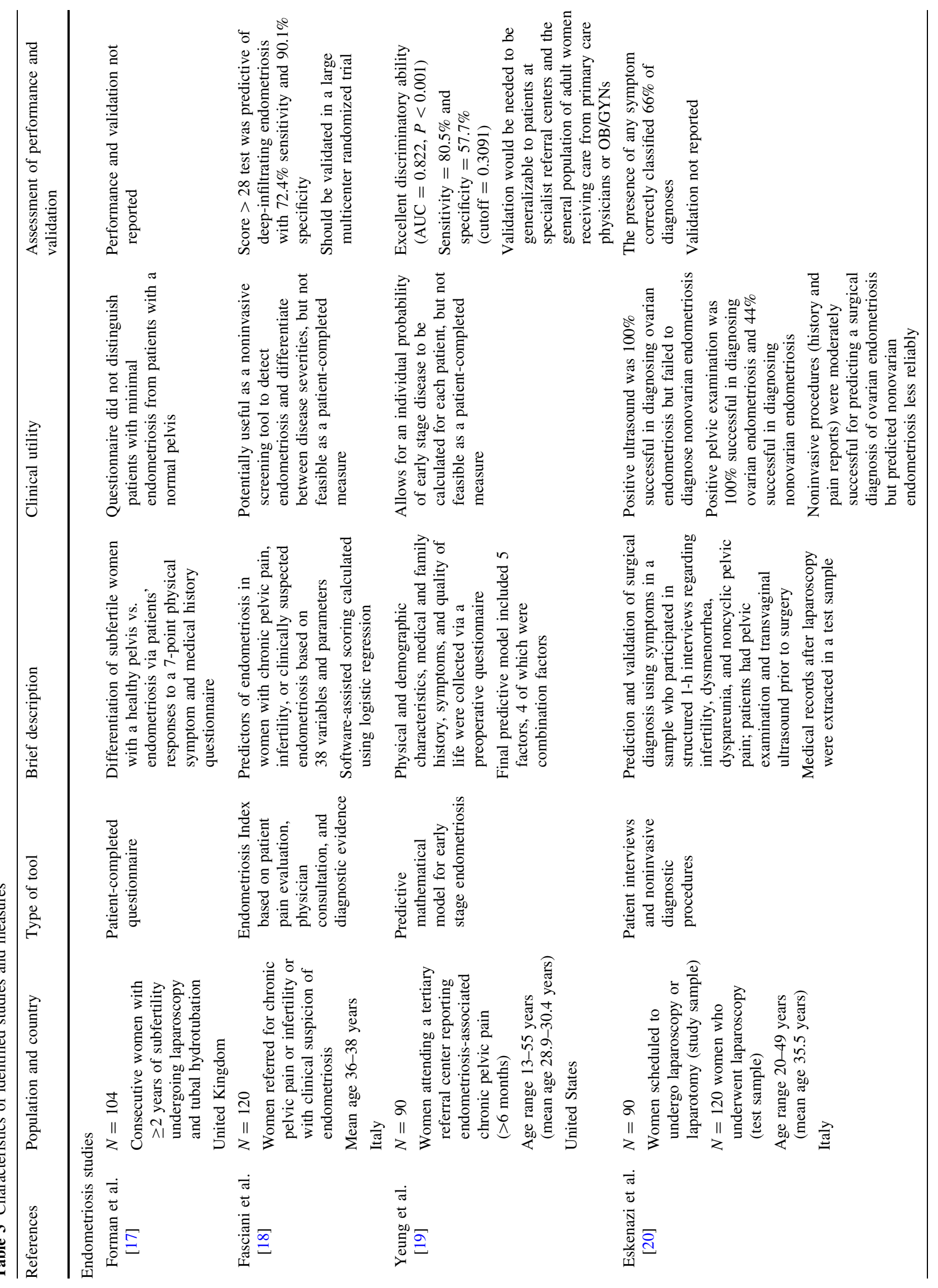




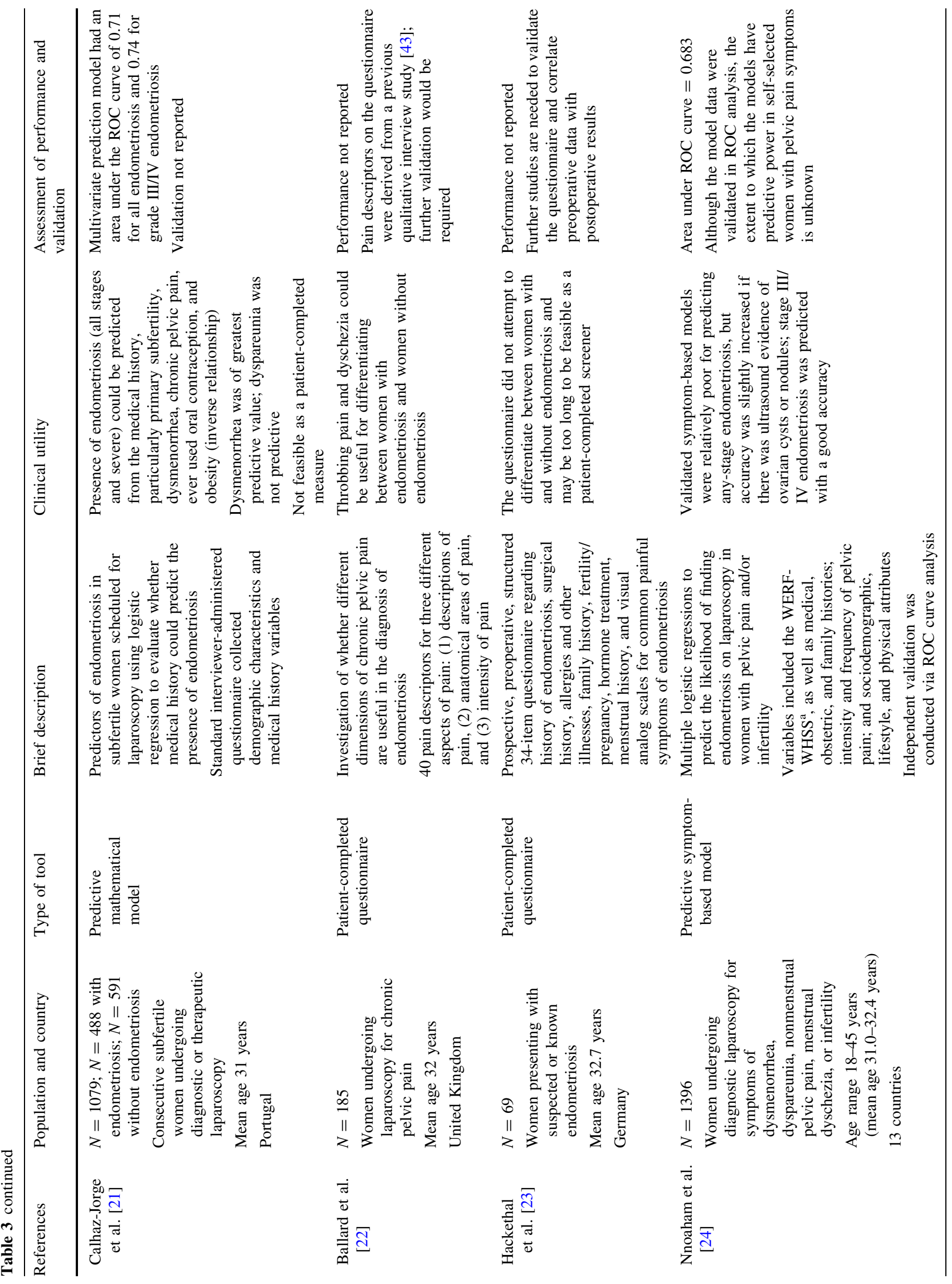




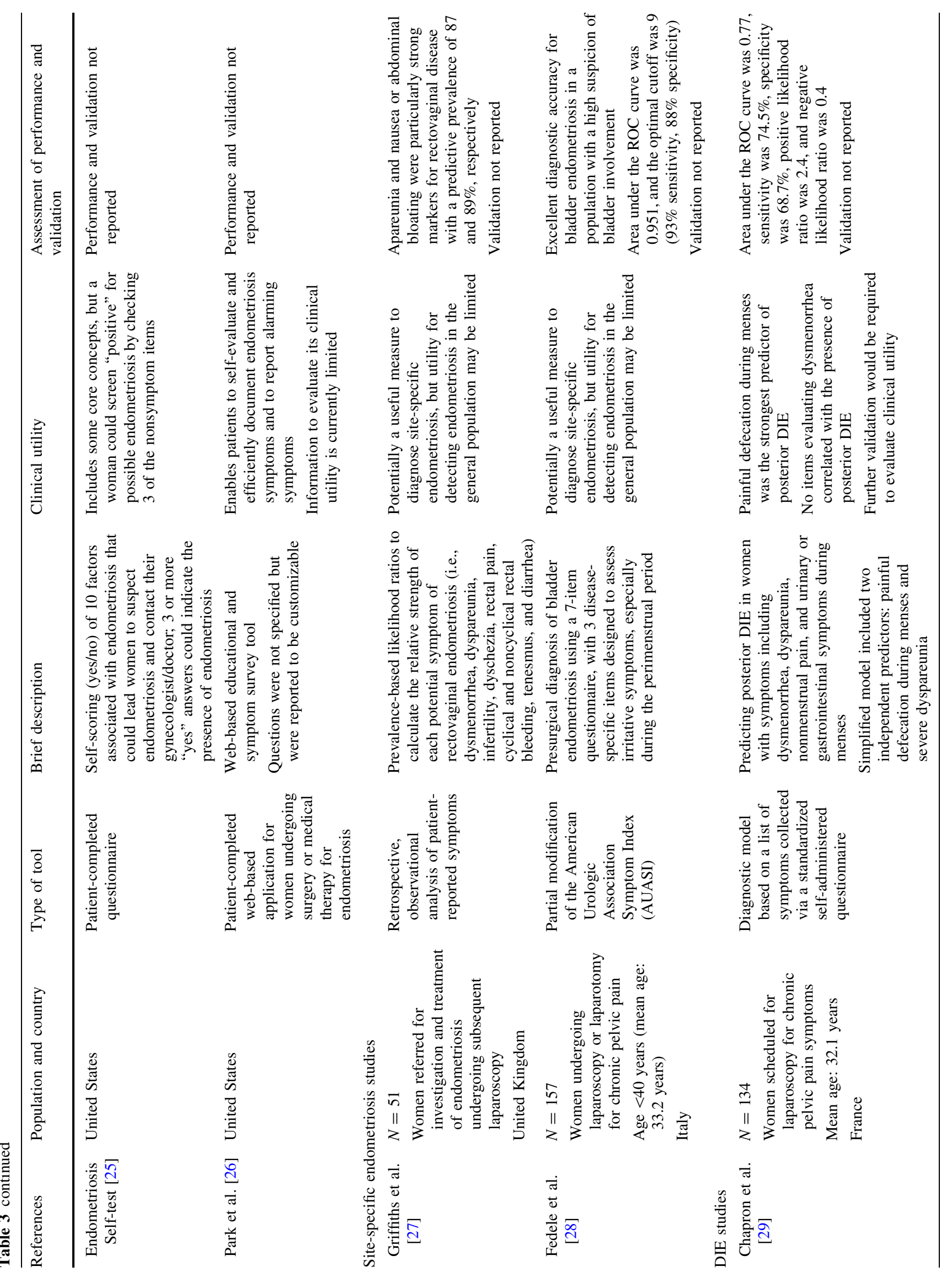




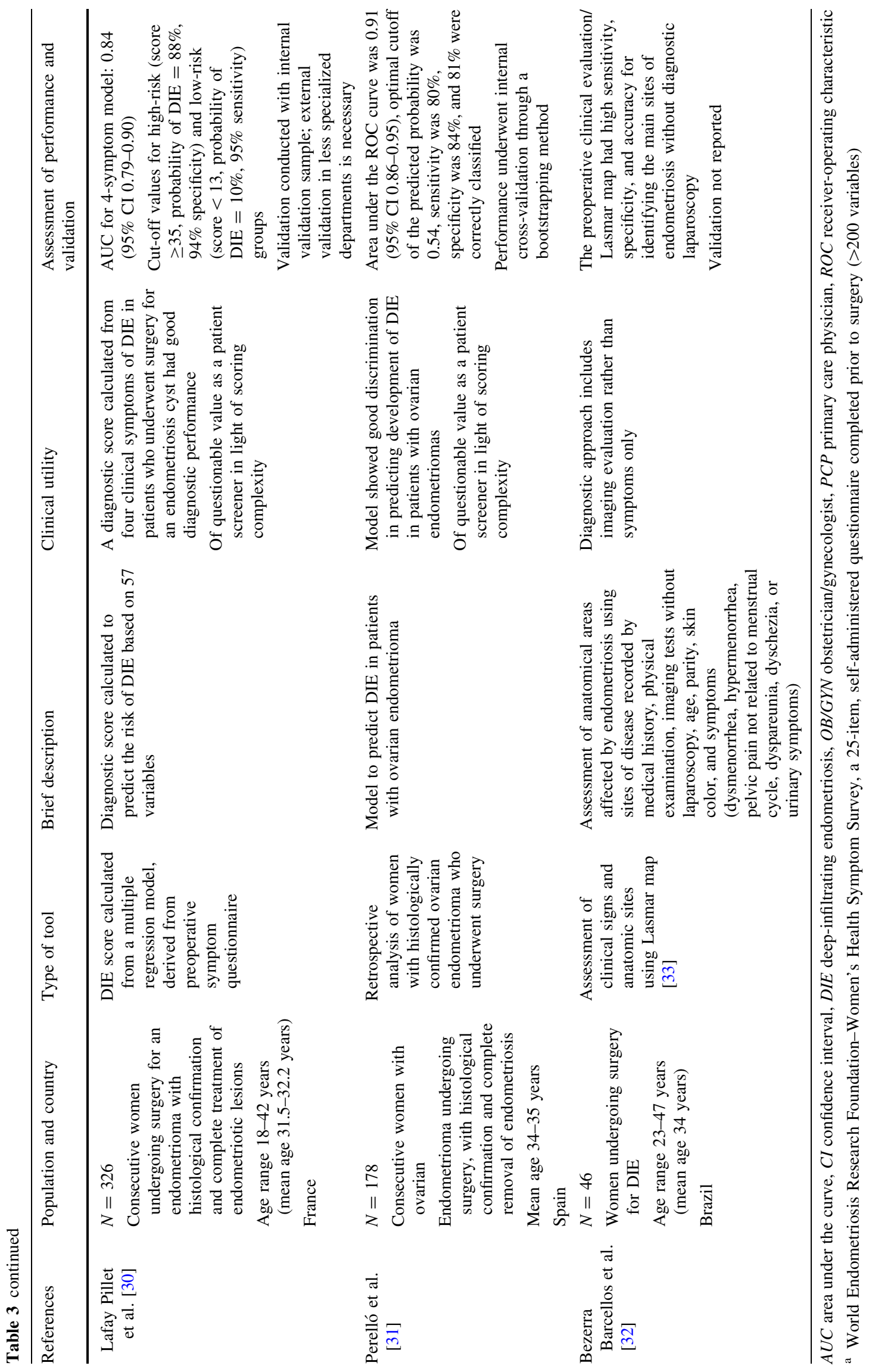


than women without endometriosis. The symptoms identified in this study could be useful for differentiating between women with endometriosis and women without endometriosis, but further validation would be required.

Hackethal et al. [23] evaluated whether a structured questionnaire, compared with retrospective review of hospital records, could improve documentation of endometriosis-specific parameters (i.e., history of endometriosis, surgical history, allergies and other illnesses, family history, fertility/pregnancy, hormone treatment, menstrual history, and visual analog scales for common painful symptoms of endometriosis) during preoperative assessment of women with suspected or confirmed endometriosis. Dysmenorrhea and dyspareunia were found to be the most common symptoms, and there was a relatively high prevalence of prior surgery for endometriosis in this population. Infertility and family history of endometriosis were not particularly common. The authors concluded that use of a structured questionnaire improved the availability of endometriosis-specific medical history in patients with known or suspected endometriosis, but the study did not attempt to differentiate between women with and without endometriosis and the questionnaire would not be practical as a self-completed screening tool owing to its length.

Finally, Nnoaham et al. [24] developed a symptombased model to predict any endometriosis, as well as stage III/IV endometriosis, in symptomatic women with no previous surgical diagnosis. Multiple logistic regression analyses were conducted, with variables including the 25-item WERF-WHSS; medical, obstetric, and family histories; intensity and frequency pelvic pain; and sociodemographic, lifestyle, and physical attributes. The models were independently validated by a receiver-operating characteristic curve analysis. Prediction of any-stage endometriosis was relatively poor but was slightly increased if there was ultrasound evidence of ovarian cysts or nodules. Stage III/IV endometriosis was predicted with good accuracy. The extent to which the models have predictive power in self-selected women with pelvic pain symptoms is unknown.

Two patient-completed tools were identified in this review, but evidence of validation or use in other published studies was not found [25, 26]. The Endometriosis Research Center self-test [25] is a 10-item questionnaire for women to self-identify potential endometriosis based on symptoms and medical history. Based on this study, women who answer "yes" to three or more questions "may have endometriosis" and are encouraged to consult a physician to discuss diagnosis and potential treatment. Although this measure includes several core concepts, women with "yes" answers to three nonsymptom questions (i.e., family history of endometriosis; miscarriage, infertility or ectopic pregnancy; autoimmune diseases; or history of pelvic surgery) could screen positive for endometriosis. For example, Park et al. [26] developed a Web-based tool for self-education and symptom documentation for women undergoing surgery or medical therapy for endometriosis. The questions are customizable and enable patients to efficiently document symptoms related to endometriosis, with a real-time interface for clinicians. In addition, participating patients are prompted to perform self-evaluations and report alarming symptoms. This tool is of potential interest, but information to evaluate its clinical utility is currently limited.

\section{Studies of endometriosis at specific sites}

Two studies evaluated measures to predict endometriosis at particular sites [27, 28]. Griffiths et al. [27] conducted a retrospective, observational analysis to assess the relative strength of a set of patient-reported symptoms (i.e., dysmenorrhea, dyspareunia, infertility, dyschezia, rectal pain, cyclical and noncyclical rectal bleeding, tenesmus, and diarrhea) in relation to rectovaginal endometriosis. Apareunia was a strong marker for rectovaginal disease and was noted by Griffiths et al. as being especially common in women with rectovaginal endometriosis, although the authors did not specify whether apareunia reflected conscious avoidance of intercourse due to concern for pain, absence of a partner, or other reasons. The absence of deep dyspareunia had a greater predictive prevalence than the presence of deep dyspareunia. Nausea or abdominal bloating was also a strong marker for rectovaginal disease; however, symptoms often attributed to irritable bowel syndrome also were common. Fedele et al. [28] developed a modified version of the American Urologic Association Symptom Index (AUASI) questionnaire for presurgical diagnosis of bladder endometriosis. Specifically, three items concerning obstructive symptoms in the standard AUASI were replaced with endometriosis-specific items designed to assess irritative symptoms, especially during the perimenstrual period. The modified AUASI demonstrated diagnostic accuracy for bladder endometriosis in a population with a high suspicion of bladder involvement. Although both of these approaches could be useful as noninvasive diagnostic tools for site-specific endometriosis, their use in the general population at risk for endometriosis is limited.

\section{Studies of deep-infiltrating endometriosis}

Four of the studies evaluated predictive measures for deepinfiltrating endometriosis specifically [29-32]. Chapron et al. [29] developed a diagnostic model based on symptoms and history, collected via a standardized self- 
administered questionnaire, to predict posterior deep-infiltrating endometriosis among women with chronic pelvic pain symptoms. The symptoms evaluated included dysmenorrhea, dyspareunia, nonmenstrual pain, and urinary and gastrointestinal symptoms during menses. Painful defecation during menses was the strongest predictor of posterior deep-infiltrating endometriosis in the model, and no items evaluating dysmenorrhea correlated with the presence of posterior deep-infiltrating endometriosis. Although the model identified symptoms that could be useful for screening for deep-infiltrating endometriosis, further validation would be required. For example, the authors stated that the diagnostic accuracy and negative predictive value likely would decrease in a population with a lower prevalence of deep-infiltrating endometriosis. Moreover, this study focused on developing a model rather than a simple screening questionnaire.

Lafay Pillet et al. [30] developed a multiple regression model, derived from a preoperative symptom questionnaire, that calculated a diagnostic score to predict the risk of deep-infiltrating endometriosis based on 57 variables (e.g., demographics, gynecologic data, history in adolescence, and characteristics of both menstrual and nonmenstrual pain). A score calculated from a set of four clinical symptoms of deep-infiltrating endometriosis (duration of pain, severe dysmenorrhea, gastrointestinal pain or dyspareunia, and infertility), showed good diagnostic performance, but the model is of questionable value as a patient screener in light of its scoring complexity. The authors noted that limitation of the study was that it was performed at a clinic specializing in deep-infiltrating endometriosis management; thus, accuracy of the score could be different in less specialized centers.

Perelló et al. [31] conducted a retrospective analysis to develop a model to predict deep-infiltrating endometriosis among women with histologically confirmed ovarian endometrioma who underwent surgery. Variables included age at first visit; BMI; the previous pregnancies, past history of surgical treatment for endometriosis, use of hormone treatment; and pain scores relating to dysmenorrhea, dyschezia, dyspareunia, and pelvic pain. The model showed good discrimination in predicting development of deep-infiltrating endometriosis in patients with ovarian endometriomas, potentially allowing prioritization for treatment at specialized referral centers. However, as with the model of Lafay Pillet et al. [30], the model of Perelló et al. [31] used complicated scoring and thus was of limited value as a patient-completed screening tool.

Finally, Bezerra Barcellos et al. [32] assessed anatomical areas affected by endometriosis using disease sites from medical history, physical examination, parity, symptoms (i.e., dysmenorrhea, hypermenorrhea, nonmenstrual pelvic pain, dyspareunia, dyschezia, and or urinary symptoms), and image evaluation [33]. The authors compared preoperative and postoperative diagnoses in patients referred for deep-infiltrating endometriosis. The preoperative clinical/ Lasmar "MAP" (i.e., a diagram to map pelvic endometriosis lesions in patients with deep-infiltrating endometriosis) evaluation had high sensitivity and specificity for identifying the main sites where endometriosis was found on laparoscopy; however, this approach to diagnosis involves imaging evaluation rather than being completely symptom-based and as such is not practical as a patient-completed screening tool.

\section{Discussion}

Diagnostic delay is a common problem in endometriosis, and identifying endometriosis as early as possible may help to avoid subsequent sequelae. The aim of this study was to identify and evaluate patient-completed, symptom-based screening tools for use in the early identification of possible endometriosis, prior to laparoscopy or without undergoing laparoscopy.

Although several measures and/or tools to predict endometriosis were identified, no patient-completed symptom-based measures that are practical for use as a screening tool in clinical practice were identified. Moreover, no follow-up studies of any measures have been published. A number of measures were administered in an interview format, relied on laboratory or physical examination variables, or otherwise required clinical input or interpretation (e.g., $[18,20,24])$. Several measures involved complex mathematical scoring, and may be of less immediate value as patient screening tools (e.g., $[19,21,24])$. Some measures demonstrated good diagnostic accuracy, but only for endometriosis at specific sites (e.g., bladder [28], deep-infiltrating endometriosis $[29,32])$. Location of a patient's endometriosis would not be the objective of a patient-driven screening questionnaire. Other measures were patient-reported and entailed simple scoring but lacked predictive accuracy. Forman et al. [17], for example, developed a 7-item patient questionnaire to identify endometriosis among subfertile women, but the measure did not successfully differentiate between women with a normal pelvis and those with endometriosis. Similarly, on the Endometriosis Self-test [25], a woman could screen "positive" for possible endometriosis by checking three of the nonsymptom items.

A recent systematic review was conducted in support of a new, not yet validated measure to identify adolescents at risk for developing endometriosis [34]. The authors of this new tool reviewed self-reported questionnaires intended to identify endometriosis in adult women and selected 
questions reported to be predictive of endometriosis, which were then included the adolescent questionnaire. Although the questionnaire includes some concepts common to other measures identified in the present review (e.g., pelvic pain and dyschezia), it also includes some concepts that are potentially specific to an adolescent population (e.g., age at first menstruation). Moreover, the questionnaire is heavily weighted on urinary symptoms, which are not a classic symptom of endometriosis. Finally, diagnosis, rather than screening, appears to be the primary application of this tool.

The previous research has characterized some symptoms associated with diagnosed endometriosis, including abdominopelvic pain, dysmenorrhea, menorrhagia, and dyspareunia $[35,36]$. However, to our knowledge, no previous literature reviews have focused on identifying and evaluating patient-completed and/or symptom-based endometriosis screening tools for adult women specifically, although other reviews have been undertaken to characterize diagnostic practices in endometriosis. A Cochrane review of noninvasive diagnostic tests for endometriosis focused largely on biomarkers (e.g., blood, urinary, and endometrial biomarkers) and diagnostic combinations (e.g., transvaginal ultrasound and physical examination) and excluded rare types of endometriosis (e.g., bladder endometriosis) [2]. The authors of that review deemed the studies to be of poor methodological quality and concluded that none of the identified diagnostic approaches were more effective than laparoscopy in diagnosing endometriosis. Another scoping review focused on clinical diagnosis of endometriosis in general (i.e., not only on symptom screeners for patients) and categorized diagnostic approaches based on whether they evaluated symptoms, signs from physical examination, or risk factors from medical history [37].

A number of endometriosis treatments are available or emerging. However, delay in diagnosis may contribute to undertreatment, continued pain, and prolonged symptom impact, which could lead to significant frustration. Helping patients to recognize their symptoms is the first step toward diagnosis and effective management of endometriosis. Patient-based screening tools empower patients with endometriosis to self-identify potential symptoms and initiate conversations with physicians about diagnosis and treatment. Specifically, there is an unmet need for instruments that can screen for endometriosis early in the course of disease, rather than at the time when a laparoscopy is scheduled to investigate the reason for pain symptoms. Patient screeners have yielded beneficial outcomes in other therapeutic areas, including neuropathic pain, psoriasis, fibromyalgia, and binge eating disorder [38-41]. The importance of engaging patients in conversations and decisions about their care is evident [42]. If patients are informed about the implications of their symptoms, physicians, in turn, may be able to optimize their care strategies to be more patient-centered.

\section{Conclusions}

A patient-completed, symptom-based screening tool for endometriosis may help patients to recognize their symptoms and engage with physicians earlier to seek a diagnosis and treatment. Existing symptom-based tools for endometriosis screening have limited clinical utility and are not fit for purpose, largely because of their length, scoring complexity, or inadequate validation. Future research should focus on developing a simple, brief patient-completed endometriosis screening tool. Ideally, such a questionnaire would be easy to score, include concepts that are important to patients, and have good predictive accuracy.

Acknowledgements Medical writing services were provided by Kate Lothman of RTI Health Solutions; this support was funded by AbbVie, Inc.

Author contributions All authors participated in data analysis and interpretation and contributed to the development of the manuscript, and all authors consented to its submission and publication. AbbVie, Inc. participated in the study design, research, data collection, analysis and interpretation, writing, review, and approval of this publication. The authors' specific contributions are as follows: ES: conceptualization, formal analysis, manuscript writing, and interpretation of study data. CMC: formal analysis, methodology, manuscript writing (lead), and interpretation of study data. AMS: conceptualization, formal analysis, funding acquisition (lead), manuscript writing, and interpretation of study data. SK: formal analysis, methodology, manuscript writing, and interpretation of study data. DBD: formal analysis, methodology, manuscript writing, and interpretation of study data. MCS: conceptualization, formal analysis, manuscript writing, and interpretation of study data.

\section{Compliance with ethical standards}

Funding This study was funded by AbbVie, Inc.

Conflict of interest This research was performed under a contract between AbbVie and RTI Health Solutions. Cathryn Carter, Shahnaz Khan, and Dana B. DiBenedetti are employees of RTI Health Solutions, funded by AbbVie to conduct this project and develop the manuscript. Ahmed M. Soliman and Michael C. Snabes are employees of AbbVie and may own AbbVie stocks/stock options. Eric Surrey has acted as a consultant and served on medical advisory boards as well as on the speakers' bureau for AbbVie.

Ethical approval This article does not contain any studies with human participants or animals performed by any of the authors.

Open Access This article is distributed under the terms of the Creative Commons Attribution 4.0 International License (http://creative commons.org/licenses/by/4.0/), which permits unrestricted use, distribution, and reproduction in any medium, provided you give appropriate credit to the original author(s) and the source, provide a link to the Creative Commons license, and indicate if changes were made. 


\section{References}

1. Johnson NP, Hummelshoj L (2013) Consensus on current management of endometriosis. Hum Reprod 28(6):1552-1568

2. Nisenblat V, Prentice L, Bossuyt PMM, Farquhar C, Hull ML, Johnson N (2016) Combination of the non-invasive tests for the diagnosis of endometriosis (review). Cochrane Database of Syst Rev 7:CD012281

3. Vigano P, Parazzini F, Somigliana E, Vercellini P (2004) Endometriosis: epidemiology and aetiological factors. Best Pract Res Clin Obstet Gynaecol 18:177-200

4. Somigliana E, Vigano P, Parazzini F, Stoppelli S, Giambattista E, Vercellini P (2006) Association between endometriosis and cancer: a comprehensive review and a critical analysis of clinical and epidemiological evidence. Gynecol Oncol 101(2): 331-341

5. Sinaii N, Cleary SD, Ballweg ML, Nieman LK, Stratton P (2002) High rates of autoimmune and endocrine disorders, fibromyalgia, chronic fatigue syndrome and atopic diseases among women with endometriosis: a survey analysis. Hum Reprod 17(10):2715-2724

6. Gao X, Outley J, Botteman M, Spalding J, Simon JA, Pashos CL (2006) Economic burden of endometriosis. Fertil Steril 86(6):1561-1572

7. Mirkin D, Murphy-Barron C, Iwasaki K (2007) Actuarial analysis of private payer administrative claims data for women with endometriosis. J Manag Care Pharm 13(3):262-272

8. Simoens S, Hummelshoj L, D'Hooghe T (2007) Endometriosis: cost estimates and methodological perspective. Hum Reprod Update 13(4):395-404

9. Soliman AM, Yang H, Du EX, Kelley C, Winkel C (2016) The direct and indirect costs associated with endometriosis: a systematic literature review. Hum Reprod 31(4):712-722

10. Fuldeore M, Yang H, Du EX, Soliman AM, Wu EQ, Winkel C (2015) Healthcare utilization and costs in women diagnosed with endometriosis before and after diagnosis: a longitudinal analysis of claims databases. Fertil Steril 103(1):163-171

11. Culley L, Law C, Hudson N, Denny E, Mitchell H, Baumgarten $M$ et al (2013) The social and psychological impact of endometriosis on women's lives: a critical narrative review. Hum Reprod Update 19(6):625-639

12. De Graaff AA, D'Hooghe TM, Dunselman GA, Dirksen CD, Hummelshoj L, WERF EndoCost Consortium, Simoens S (2013) The significant effect of endometriosis on physical, mental and social wellbeing: results from an international cross-sectional survey. Hum Reprod 28(10):2677-2685

13. Greene R, Stratton P, Cleary SD, Ballweg ML, Sinaii N (2009) Diagnostic experience among 4,334 women reporting surgically diagnosed endometriosis. Fertil Steril 91(1):32-39

14. Matsuzaki S, Canis M, Pouly JL, Rabischong B, Botchorishvili R, Mage G (2006) Relationship between delay of surgical diagnosis and severity of disease in patients with symptomatic deep infiltrating endometriosis. Fertil Steril 86:1314-1316

15. Kennedy S, Bergqvist A, Chapron C, D'Hooghe T, Dunselman G, Greb R, ESHRE Special Interest Group for Endometriosis and Endometrium Guideline Development Group et al (2005) ESHRE guideline for the diagnosis and treatment of endometriosis. Hum Reprod 20(10):2698-2704

16. Apostolopoulos NV, Alexandraki KI, Gorry A, Coker A (2016) Association between chronic pelvic pain symptoms and the presence of endometriosis. Arch Gynecol Obstet 293(2):439-445

17. Forman RG, Robinson JN, Mehta Z, Barlow DH (1993) Patient history as a simple predictor of pelvic pathology in subfertile women. Hum Reprod 8(1):53-55

18. Fasciani A, Repetti F, Binda GA, Puntoni M, Meroni MG, Bocci $G$ (2010) Endometriosis index: a software-derived score to predict the presence and severity of the disease. J Endometriosis 2(2):79-86

19. Yeung P, Bazinet C, Gavard JA (2014) Development of a symptom-based, screening tool for early-stage endometriosis in patients with chronic pelvic pain. J Endometriosis 6(4):174-189

20. Eskenazi B, Warner M, Bonsignore L, Olive D, Samuels S, Vercellini P (2001) Validation study of nonsurgical diagnosis of endometriosis. Fertil Steril 76(5):929-935

21. Calhaz-Jorge C, Mol BW, Nunes J, Costa AP (2004) Clinical predictive factors for endometriosis in a Portuguese infertile population. Hum Reprod 19(9):2126-2131

22. Ballard K, Lane H, Hudelist G, Banerjee S, Wright J (2010) Can specific pain symptoms help in the diagnosis of endometriosis? A cohort study of women with chronic pelvic pain. Fertil Steril 94(1):20-27. doi:10.1016/j.fertnstert.2009.01.164 (Epub 2009 Apr 1)

23. Hackethal A, Luck C, von Hobe AK, Eskef K, Oehmke F, Konrad L (2011) A structured questionnaire improves preoperative assessment of endometriosis patients: a retrospective analysis and prospective trial. Arch Gynecol Obstet 284(5):1179-1188

24. Nnoaham KE, Hummelshoj L, Kennedy SH, Jenkinson C, Zondervan KT, World Endometriosis Research Foundation Women's Health Symptom Survey Consortium (2012) Developing symptom-based predictive models of endometriosis as a clinical screening tool: results from a multicenter study. Fertil Steril 98(3):692-701.e5

25. Endometriosis Research Center (2012) Endometriosis screening and education kit: empowering materials for understanding and living well in spite of endometriosis. http://selahfunrunners.wee bly.com/uploads/2/6/4/0/2640301/2012screeningeducationkit. pdf. Accessed 3 May 2016

26. Park et al (2014) Development and assessment of a web-based software application to facilitate self-education and symptom documentation in women undergoing surgery or medical therapy for endometriosis. World Congress of Endometriosis (abstract P-242)

27. Griffiths AN, Koutsouridou RN, Penketh RJ (2007) Predicting the presence of rectovaginal endometriosis from the clinical history: a retrospective observational study. J Obstet Gynaecol 27(5):493-495

28. Fedele L, Bianchi S, Carmignani L, Berlanda N, Fontana E, Frontino G (2007) Evaluation of a new questionnaire for the presurgical diagnosis of bladder endometriosis. Hum Reprod 22(10):2698-2701

29. Chapron C, Barakat H, Fritel X, Dubuisson JB, Bréart G, Fauconnier A (2005) Presurgical diagnosis of posterior deep infiltrating endometriosis based on a standardized questionnaire. Hum Reprod 20(2):507-513

30. Lafay Pillet MC, Huchon C, Santulli P, Borghese B, Chapron C, Fauconnier A (2014) A clinical score can predict associated deep infiltrating endometriosis before surgery for an endometrioma. Hum Reprod 29(8):1666-1676

31. Perelló M, Martínez-Zamora MA, Torres X, Munrós J, Llecha S, De Lazzari E et al (2017) Markers of deep infiltrating endometriosis in patients with ovarian endometrioma: a predictive model. Eur J Obstet Gynecol Reprod Biol 209:55-60

32. Bezerra Barcellos M, Lasmar B, Lasmar R (2016) Agreement between the preoperative findings and the operative diagnosis in patients with deep endometriosis. Arch Gynecol Obstet 293:845-850

33. Lasmar RB, Lasmar BP, Pillar C (2012) Diagram to map the locations of endometriosis. Int J Gynaecol Obstet 118(1):42-46

34. Geysenbergh B, Dancet EA, D'Hooghe T (2016) Detecting endometriosis in adolescents: why not start from self-report screening questionnaires for adult women? Gynecol Obstet Invest. doi: 10.1159/000452098

35. Ballard KD, Seaman HE, de Vries CS, Wright JT (2008) Can symptomatology help in the diagnosis of endometriosis? Findings 
from a national case-control study-Part 1. BJOG 115(11): 1382-1391

36. Fuldeore MJ, Soliman AM (2016) Prevalence and symptomatic burden of diagnosed endometriosis in the United States: national estimates from a cross-sectional survey of 59,411 women. Gynecol Obstet Invest. doi:10.1159/000452660

37. Riazi H, Tehranian N, Ziaei S, Mohammadi E, Hajizadeh E, Montazeri A (2015) Clinical diagnosis of pelvic endometriosis: a scoping review. BMC Womens Health 15:39. doi:10.1186/ s12905-015-0196-Z

38. Portenoy R (2006) Development and testing of a neuropathic pain screening questionnaire: ID Pain. Curr Med Res Opin 22(8):1555-1565

39. Brown TM, Zhao Y, Haydysch, E, Martin S, Liu J, Tran MH (2014) Development of a psoriasis patient-reported tool to facilitate patient-doctor discussion. In: Poster presented at the Dermatology Nurses Association 32nd annual convention, Orlando
40. Martin SA, Coon CD, McLeod LD, Chandran A, Arnold LM (2014) Evaluation of the fibromyalgia diagnostic screen in clinical practice. J Eval Clin Pract 20(2):158-165

41. Herman BK, Deal LS, DiBenedetti DB, Nelson L, Fehnel SE, Brown TM (2015) Development of the binge-eating disorder screener. Poster presented at the American Psychiatric Association Annual Meeting, Toronto

42. Food and Drug Administration (FDA) (January 2016) Food and Drug Administration Safety and Innovation Act (FDASIA). Section 1137: patient participation in medical product discussions-report on stakeholder views. http://www.fda.gov/down loads/forpatients/about/ucm486859.pdf. Accessed 13 June 2016

43. Ballard K, Lowton K, Wright J (2006) What's the delay? A qualitative study of women's experiences of reaching a diagnosis of endometriosis. Fertil Steril 86(5):1296-1301 